\title{
BMJ Open Protocol for a prospective, observational, hospital-based multicentre study of nosocomial SARS-CoV-2 transmission: NOSO-COR Project
}

\author{
Mitra Saadatian-Elahi (1) ,1,2 Valentina Picot, ${ }^{1,3}$ Laetitia Hénaff, ${ }^{1}$ \\ Florence K Pradel, ${ }^{1,3}$ Vanessa Escuret, ${ }^{4,5}$ Cédric Dananché, ${ }^{1,2}$ Christelle Elias, ${ }^{1,2}$ \\ Hubert P Endtz, ${ }^{1,3}$ Philippe Vanhems ${ }^{1,2,6}$
}

To cite: Saadatian-Elahi M, Picot V, Hénaff L, et al. Protocol for a prospective, observational, hospital-based multicentre study of nosocomial SARS-CoV-2 transmission: NOSO-COR Project. BMJ Open 2020;10:e039088. doi:10.1136/ bmjopen-2020-039088

- Prepublication history and additional material for this paper are available online. To view these files, please visit the journal online (http://dx.doi. org/10.1136/bmjopen-2020039088).

Received 06 April 2020 Revised 28 July 2020

Accepted 24 September 2020

Check for updates

(c) Author(s) (or their employer(s)) 2020. Re-use permitted under CC BY-NC. No commercial re-use. See rights and permissions. Published by BMJ.

For numbered affiliations see end of article.

Correspondence to Dr Philippe Vanhems; philippe.vanhems@chu-lyon.fr

\section{ABSTRACT}

Introduction The newly identified SARS-CoV-2 can cause serious acute respiratory infections such as pneumonia. In France, mortality rate in the general population was approximately $10 \%$ and could reach higher levels at the hospital. In the current context of high incidence rates of SARS-CoV-2 in the community, a significant increase in the rate of nosocomial transmission is expected. The risk of nosocomial transmission could even be higher in lowincome countries that have fragile healthcare systems. This protocol is intended to estimate the prevalence and incidence of suspected or confirmed cases of nosocomial SARS-CoV-2 infection, the clinical spectrum and the determinants (risk factors/protective) at participating hospitals.

Methods and analysis This will be an international multicentre prospective, observational, hospital-based study in adults and children. It will include volunteer patients and healthcare professionals in France and hospitals affiliated with the GABRIEL network. Demographic and clinical data will be collected using case report forms designed especially for the purpose of the project. A nasopharyngeal swab will be collected and tested for SARS-CoV-2 by reverse-transcriptase PCR. Characteristics of the study participants, the proportion of confirmed nosocomial SARS-CoV-2 infections relative to all patients with syndromes suggestive of SARS-CoV-2 infection, will be analysed. Appropriate multivariate modelling will be used to identify the determinants associated with nosocomial onset.

Ethics and dissemination This study was approved by the clinical research and committee of all participating countries. The findings will be submitted to peer-reviewed journal for publication and shared with national health authorities.

Trial registration number NCT04290780.

\section{INTRODUCTION}

Coronaviruses are enveloped viruses that mainly infect the upper digestive and respiratory tracts of mammals and birds. In humans, the viruses can cause mild respiratory infections but can also lead to serious infections
Strengths and limitations of this study

- This prospective study will generate original data on nosocomial SARS-CoV-2 infection in France and in low-income countries.

- The results will provide the opportunity to document nosocomial SARS-CoV-2 infection and pave the way to set up new preventive recommendations.

- Selection bias owing to access to care in different populations and bias owing to the extent of access to personal protective equipment, in particular, in low-income countries may occur.

- The non-exhaustivity of the confounders to be collected should be considered for interpretation of the results.

- Only symptomatic cases were included in the study. Paucisymptomatic and asymptomatic carriers might have been missed.

such as pneumonia. During the past decade, two human coronaviruses, SARS-CoV and Middle East respiratory syndrome coronavirus (MERS-CoV), were the source of severe acute respiratory syndrome (SARS) and Middle East respiratory syndrome epidemics in 2002 and 2012, respectively. ${ }^{1}$

The newly identified SARS-CoV-2 is a singlestranded RNA virus belonging to the coronavirus crown virus family of the subfamily Orthocoronaviridae. SARS-CoV-2 appears to be a recombinant virus between the bat coronavirus and a coronavirus of unknown origin. ${ }^{2}$ The virus was first detected in December 2019 in Hubei province of China ${ }^{3-5}$ and spread widely throughout China before crossing the borders into other countries. ${ }^{6}$ SARS-CoV-2 is mainly transmitted by respiratory droplets but can also be spread through aerial droplets or fomites by contact. ${ }^{7}$

COVID-19 is the emerging infectious disease caused by SARS-COV-2 infection. 
COVID-19 can manifest in many ways, ${ }^{8-10}$ even if asymptomatic cases have also been reported. ${ }^{11}$ In France, a mortality rate of $10 \%$ has been reported in the general population. ${ }^{12}$ The estimated median incubation period of COVID-19 was 5.1 days (CI 4.5 to 5.8 days) in a pooled analysis of 181 confirmed cases reported from around the world. $^{13}$

On 11 March 2020, the WHO declared COVID-19 a pandemic, pointing to over 118000 cases of coronavirus in over 110 countries and territories around the world with the sustained risk of further global spread.

Analysis of the clinical presentation of the first case series $(n=41)$ showed that $31 \%$ of the patients were admitted to intensive care units (ICUs) and the crude mortality rate was $15 \% .^{14}$ COVID-19-related complications, including severe pneumonia, respiratory distress, secondary bacterial infection or decompensation for chronic heart or respiratory disease, mainly affected patients over 65 years. The cases described to date mostly occurred among patients with a history of chronic pathology, ${ }^{4}$ who were therefore more likely to be hospitalised.

In the absence of a preventive vaccine or curative treatment, current efforts to prevent and control the spread of SARS-CoV-2 are based on early detection of cases, along with infection control measures such as precautions against respiratory and direct-contact spread. In addition, specific measures are being applied to patients who should be cared for in single rooms: negative pressure ventilation, if possible; wearing surgical masks; and practising strict hand hygiene using hydroalcoholic solutions, $70 \%$ ethanol solutions or chlorine-containing disinfectants. Class 2 or 3 or N95 filtering facepiece respirators are recommended when performing aerosol-generating procedures. Specific recommendations have also been issued from the WHO and Centers for Disease Control and Prevention. ${ }^{15} 16$

As for the SARS and MERS-CoV epidemics and other respiratory viruses such as influenza or respiratory syncytial virus (RSV), cases of intrahospital transmission of SARS-CoV-2 have been reported and are going to continue to occur. In Wuhan alone, 1080 healthcare professionals (HCPs) were infected. ${ }^{17}$ In China, more than 3300 HCPs were infected as of early March, and in Italy, 20\% of the HCPs participating in a survey reported COVID-19 infections. ${ }^{18}$ According to the National French Public Health Agency, Santé Publique France, more than 30000 HCPs were infected since March $2020 .^{19}$

HCPs have a key position in the transmission process because they are exposed to both community-acquired and nosocomial cases. ${ }^{20} 21$ This risk is amplified when the incidence of the infection in the community is high. In the current context of high incidence rates of SARS-CoV-2 in the community, a significant increase in the rate of nosocomial transmission is therefore expected. The risk of nosocomial transmission would be even higher in lowincome countries, owing to several factors such as the delay in diagnosis of patients with COVID-19 and the lack of infrastructure, trained personnel, isolation units and infection control programmes.

Beyond the morbidity and mortality associated with nosocomial COVID-19 infection, the impact on the organisation of care and the additional costs caused by longer hospital stays are still unknown but will certainly be of consequence.

The link between hospital (nosocomial) and community attack rates is a good indicator of the effect of hospitalisation on the transmission of respiratory viruses. ${ }^{22}$ Attack rate is defined as the proportion of infected patients among the total number of patients at risk of being infected during the epidemic period. The denominator, that is, the total number of at-risk individuals in the community, is provided by the regional agency of public health for the calculation of attack rates in the community, while the number of hospitalised patients or patientdays of hospitalisation will be used as denominator for in-hospital calculation. Better understanding of the transmission chains of SARS-CoV-2 and the impact of control measures in healthcare units is essential to achieving control of the pandemic. For example, the configuration of care units also appears to play a role in transmission. Indeed, hospitalisation in a double room increased the risk of contracting influenza in a hospital by 2.67 -fold. ${ }^{23}$

Implementation of appropriate hygiene and preventive measures play a decisive role in the control of nosocomial risk. Countries have implemented various measures according to their national guidelines to curb the propagation of this emergent virus; thus, their comparison could allow identification of possible areas for improvement in patient care. This article describes the protocol of an international multicentre prospective study whose aims are to document suspected or confirmed nosocomial cases of COVID-19, their clinical spectrum and the prognostic factors at participating hospitals.

\section{Objectives}

The principal aim of this study was to estimate the prevalence and incidence of suspected or confirmed cases of nosocomial SARS-CoV-2 infection among HCPs and patients.

The secondary objectives were to (1) to describe and document cases of community-acquired SARS-CoV-2 infection (prevalent cases on hospital admission) likely to be the source of nosocomial infection and the clinical spectrum; (2) describe and document hospital-acquired SARS-CoV-2 infection (incident cases) that may be the source of nosocomial transmission and their clinical spectrum; (3) describe observance of infection control practices in settings where nosocomial transmission could occur and compare the attack rates between the hospital and the community; (4) estimate the incidence of infectious syndromes attributed to SARS-CoV-2 and the proportion of severe cases, including deaths; (5) describe subpopulations with SARS-CoV-2 infection, depending on the hospital ward (eg, internal medicine vs surgery vs ICUs); (6) compare the adjusted attack rates of 


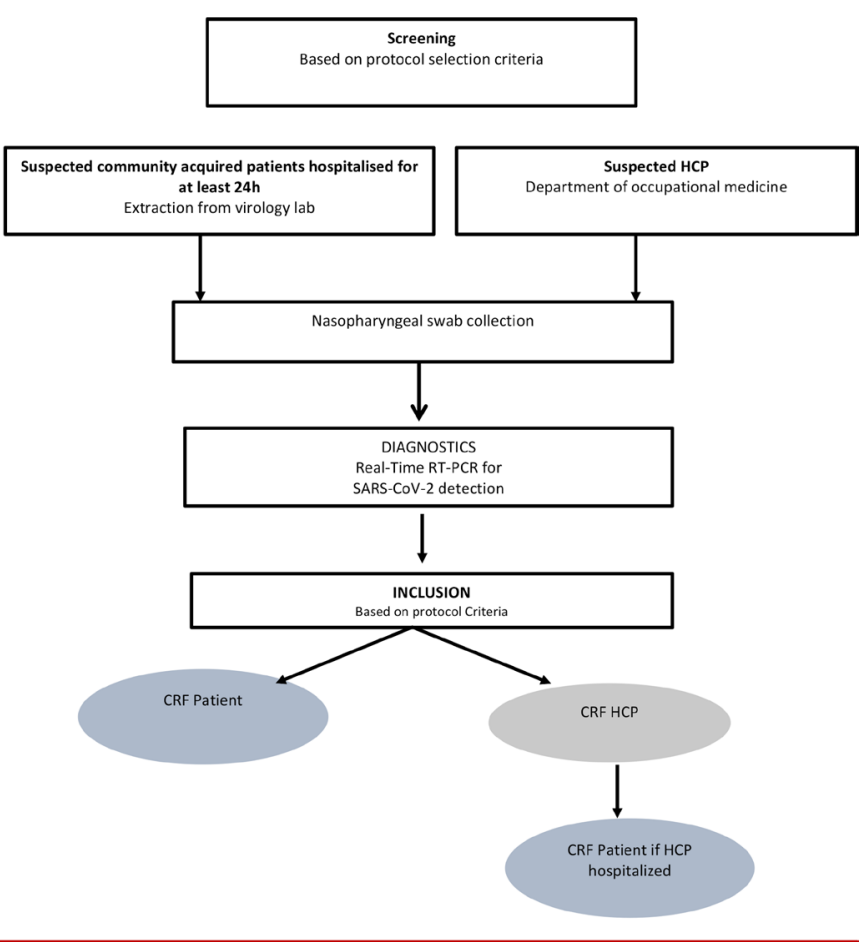

Surveillance of hospital wards for the Identification of secondary cases

Figure 1 Study flowchart. CRF, case report form; HCP, healthcare professional; RT-PCR, reverse-transcription PCR.

nosocomial and community-acquired SARS-CoV-2 infection to identify contextual and/or environmental protective and risk factors in both the hospital and community setting; and (7) calculate the crude mortality rate and adjusted rates according to clinical features stratified by age, comorbidities, type of ward and community versus hospital-acquired infection.

\section{METHODS}

\section{Study design and setting}

This prospective, observational, hospital-based study will be carried out among volunteer patients and HCPs in university-affiliated hospitals (Hospices Civils de Lyon, Lyon, France), eight volunteered hospitals across France (Dijon, Grenoble, Paris, Puy-en-Velay, Saint Etienne, Périgueux, Eaubonne and Suresnes) and hospitals affiliated with the GABRIEL network (https://www.gabrielnetwork.org/), a network of research institutions mainly located in low-income countries and focused on the aetiological agents of pneumonia. Other French or European university hospitals will be welcome and able to join the project on a voluntary basis.

\section{Patient and public involvement}

No patients were involved in our study.

\section{Recruitment}

\section{Inclusion criteria}

Any adult/child patient or HCP from participating hospitals who gives oral or written informed consent and who presents an infectious syndrome based on the WHO definition of COVID- $19^{24}$ are included in the study:

Suspected case: a patient with acute respiratory illness (fever and at least one sign/symptom of respiratory disease, eg, cough or shortness of breath).

A patient with any acute respiratory illness and having been in contact with a confirmed or probable COVID-19 case (household, professional activity or travel) in the last 14 days prior to symptom onset.

A patient with severe acute respiratory illness (fever and at least one sign/symptom of respiratory disease, eg, cough or shortness of breath, and requiring hospitalisation) and in the absence of an alternative diagnosis that fully explains the clinical presentation.

Confirmed case: a person with laboratory confirmation of COVID-19 infection.

\section{Exclusion criteria}

Individuals who do not meet the aforementioned criteria were excluded from the study.

The study flowchart is shown in figure 1. Eligible patients will be identified by a clinical research assistant who will regularly contact hospital wards (emergency, geriatric and infectious diseases) and will review the results of the virology laboratory. The clinical research assistant will meet eligible individuals to explain the purpose of the study and to obtain written informed consent. Nosocomial cases will be defined according to the SARS-CoV-2 incubation period, estimated on average at 5 days and ranging from 1 to 14 days. ${ }^{13}$ We assumed that a delay greater than 48 hours between hospital admission and the onset of symptoms could be used to define a nosocomial case in order to ensure high sensitivity.

Identification of infected HCPs will be based on data from the department of occupational medicine. A confidential interview with the symptomatic HCP will be organised to describe the purpose of the study and to obtain written informed consent.

\section{Participant timeline}

Enrolled participants will be followed up during their entire hospital stay. Information on further complications that occurred during the follow-up period and vital status will be collected from the patient's medical file.

\section{Index case}

An index case is defined as the first RT-PCR confirmed case of SARS-CoV-2 infection in a given department during a given period.

\section{Secondary case}

A secondary case is defined as an RT-PCR-confirmed case (1) who was in contact with an index case during the contagious period, currently defined as 5 days before and 15 days after the onset of symptoms in the index case; (2) who developed clinical features compatible with the diagnostic criteria of SARS-CoV-2 infection within 15 days of the onset of symptoms in the index case. 
The period of detection of secondary cases will be calculated according to the period of contagiousness of the index case: (date of onset of symptoms) to (date of onset of symptoms +15 days) and the incubation period of secondary cases (1-15 days). Secondary cases will therefore be clinically detected in the time interval between (start date of symptoms in the index case +1 day) to (start date of symptoms in the index case +15 days).

\section{Data collection \\ Clinical data}

Data will be collected using case report forms (CRFs) designed especially for the purpose of this project for patients and HCPs (online supplemental data). According to the operating modes of each participating hospital and their organisation in terms of clinical or epidemiological research, the CRF will be completed during a face-to-face interview and the use of medical records. The CRF has been pretested on a small number of patients and HCPs and adjusted accordingly.

CRF will include demographic characteristics, underlying comorbidities, medical history, and clinical, biological and laboratory data on the SARS-CoV-2 infectious episode. In case the HCP is hospitalised, a patient's CRF including additional data regarding information on hospitalisation (ward specialty and type of room) and biological parameters (blood cell counts) will be completed.

The characteristics of the hospital and participating wards (specialties, number of beds, number of nurses and doctors, and presence of an infection control unit) and infection control policies regarding the risk of infection by SARS-CoV-2 will also be collected (online supplemental data). In addition, all participating centres are requested to provide a copy of their guidelines regarding COVID-19 preventive measures and the adjustments of the guideline over the epidemic period.

\section{Biological sample collection and testing}

A nasopharyngeal swab will be collected and tested for the presence of SARS-CoV-2 RNA by real-time reversetranscription PCR (RT-PCR) for each patient or HCP that meets inclusion criteria. Samples will be tested by the closest virology laboratory where state-of-the-art SARS-CoV-2 assay is performed. The results will be reported on laboratory forms created specifically for this study.

If feasible, RT-PCR will be performed to detect a panel of other respiratory viruses (influenza A and B, RSV, rhinovirus and metapneumovirus), depending on the diagnostic practices available at each participating centre. Furthermore, SARS-CoV-2 sequencing will be performed, depending on the technical platforms available.

Biobanks are expected to be constituted and, if possible, an aliquot of each sample will be stored in a Micronic tube at $-20^{\circ} \mathrm{C}$ for at least 5 years after written informed consent is obtained.

\section{Statistical methods}

Sample size

Given the descriptive nature and surveillance objectives of this observational study, reaching a predefined number of subjects is not realistic.

\section{Statistical analyses}

Data will be analysed using statistical methods to describe the characteristics of the healthcare setting and study participants, the percentage of COVID-19 of those presenting influenza-like illnesses, the percentage of patients with nosocomial COVID-19 among the confirmed cases.

The primary criteria will be the proportion of patients and HCPs with confirmed nosocomial SARS-CoV-2 infection relative to all patients and HCPs with syndromes suggestive of SARS-CoV-2 infection during the study period.

Secondary outcome criteria will be to (1) describe the delay of onset of suspected or confirmed nosocomial COVID-19 infection for hospitalised patients, (2) estimate the attack rate of confirmed COVID-19 cases in hospitalised patients according to their length of stay and (3) estimate the attack rate of confirmed COVID-19 cases among HCPs.

Categorical variables will be described with frequencies $(\%)$ and compared using $\chi^{2}$ or Fisher exact test as appropriate. Continuous variables will be described using mean and SD or median and IQR according to normal distribution, and compared using Mann-Whitney or KruskalWallis test as appropriate. Relative risk and 95\% CI will be used as a measure of association. Statistical tests were two-tailed with a level of statistical significance of $<0.05$.

Appropriate multivariate modelling methods will be applied, depending on the numbers of patients and the hypothesis tested (Poisson regression, Cox regression and logistic regression) to identify the determinants independently associated with outcomes. For example, the length of stay will be considered as the duration of exposure.

\section{Data management and archiving Case report form}

All required information will be recorded on a paperbased CRF in a clear and legible manner, and justification must be provided for all missing data. Erroneous data noted in the CRF will be clearly crossed out and the correct data will be written next to the crossed-out information, accompanied by the initials of the investigator or authorised person who made the correction, the date and, if possible, a justification for the correction.

To reduce data-entry errors by predefining plausibility checks and facilitate the rapid transfer of data, the electronic case report form (e-CRF) version of the study CRF will also be available.

Transfer of the data to the coordinating centre in Lyon (France) will be performed via the e-CRF following approval by the French Commission nationale de 
l'informatique et des libertés-National Commission for Data Protection. An alternative possibility will be to ensure periodic reporting (every 10 inclusions) by e-mailing the scan of the completed CRF to the coordinating centre.

\section{Data management}

Collected data will be computerised in the coordinating centre in Lyon, France. Source documents and databases will be anonymous and locked with a password known only to the scientific staff. These data will be kept for a minimum of 10 years after the end of the study.

\section{Archiving}

According to French law, the sponsor will keep the study documents (protocol and annexes, possible amendments, information forms, CRF, statistical analysis plan and output, and the final study report) for a minimum of 25 years. After this period, the sponsor will be consulted before any data are destroyed.

Study-related documents and reports may be subject to audit or inspection by the sponsor and/or other authorised bodies.

This study is part of the 'reference methodology' (MR-003) in application of article 54, paragraph 5, of French law No. 78-17 of 6 January 1978. This change was approved on 5 January 2006 and modified on 21 July 2016. The Hospices Civils de Lyon, the promoter of the study, has signed a commitment to comply with this reference methodology.

\section{Rights to access source documents}

Source documents are defined as any original documents, data and records in which data collected for a clinical trial are first recorded. Source documents will be kept by the investigator for 10 years or, if hospital medical records, by the hospital for 10 years. Each centre will have access to its own data during the study.

To ensure quality control and auditing, the sponsor will be responsible for obtaining the agreement of all parties involved in the research to guarantee direct access to all places where the research is carried out and to source data, documents and reports.

In accordance with current laws and regulations (articles L.1121-3 and R.5121-13 of the French Public Health Code), all documents and personal data required for monitoring, quality control or auditing will be available to the persons in charge of these activities.

\section{Confidentiality}

The principal and associate investigators are required to respect professional confidentiality (articles 226-13 and 226-14 of the French Penal Code). In accordance with French laws regarding the confidentiality of study participant personal data (article L.1121-3 of the French Public Health Code) and clinical/laboratory results obtained throughout the study (article R. 5121-13 of the French Public Health Code), individuals with direct access to the data will take all necessary precautions to ensure the confidentiality of the overall collected information.
All personal data concerning study participants will remain strictly confidential. To respect privacy, all participant details will be anonymous for the purpose of database preparation. Study subjects will be coded as follows: AAXXXXZZYY. The first two letters will be either PA for a patient and HP for HCPs. The following four digits will depend on the order of inclusion of the case, and the next two letters will correspond to the hospital code. Finally, the last two letters will correspond to the country in which the hospital is located.

\section{Quality control and assurance}

Quality assurance audits will be carried out by persons appointed by the promoter, as well as the inspections carried out by competent authorities. All data, documents and reports can be subject to regulatory audits and inspections without hindering medical confidentiality.

\section{ETHICS AND DISSEMINATION \\ Ethical approval}

Ethical approval has been obtained from all participating hospitals as follows: France (clinical research and committee of Ile de France V, 8 March 2020); Guinea (Comité National d'éthique pour la recherche en santé, 20 April 2020); Mali (Comité d'Ethique des Facultés de Médecine et de Pharmacie, 16 April 2020); Ivory coast (Comité National d'Ethique des Sciences et de la SantéMI la Recherche en Sant é, 10 April 2020); Madagascar (Comité National d'Ethique de la Recherche Biomédicale, 30 March 2020); Bangladesh (ICCDR,B ethical committee, 10 April 2020); Lebanon (USJ Hôtel Dieux comité d'éthique, 5 March 2020) and Brazil (Comitês ee Ética em Pesquisas, 28 May 2020).

\section{Informed consent}

Patients and HCPs will be informed of the objectives and their rights to refuse to participate in the study or withdraw at any time using simple, understandable terms. This information will be provided by an information and consent form given to each participant. According to French law, voluntary, oral informed consent will be obtained by the investigator before inclusion for the epidemiological data, while signed consent is needed for the collection of biological banks. Consenting will be performed following the country's ethical guidelines. Informed consent will be obtained from the parents of included children $(<18$ years old $)$.

\section{Regulatory compliance}

The research will be conducted in accordance with applicable laws and regulations currently in place in France and internationally.

\section{Withdrawal criteria}

Subjects may request to withdraw from the study at any time and for any reason without having to justify. In the event of a premature withdrawal, the investigator must 
document the participant's reasons for withdrawal as completely as possible.

\section{Stopping the research study}

The sponsors reserve the right to interrupt the study at any time if the objectives are not being met. In the event of premature termination of the study for security reasons, the information will be transmitted by the sponsors to all concerned parties and to the local ethical committee within 15 days.

\section{Protocol amendments}

In the eventuality of changes in the existing protocol that significantly affect the scope or the scientific quality of the investigation, an amendment containing a verbatim description of the changes and reference (date and number) to the submission that contained the original protocol will be submitted to the ethical committee for their approval.

\section{Dissemination}

Communications and scientific reports that emerge from this study will be carried out under the responsibility of the principal investigator in agreement with the associated investigators. Publication rules will follow international recommendations. ${ }^{25}$ The findings will also be shared with national health authorities.

Authorship will follow the guidelines established by the International Committee of Medical Journal Editors (http://www.icmje.org/), which require substantive contributions to the design, conduct, interpretation and reporting of a trial.

\section{DISCUSSION}

Human-to-human transmission of SARS-CoV-2, including nosocomial transmission, is now well documented. The risk of amplification of spread of the disease in healthcare facilities is strong in case of a lack of infection control measures. Early recognition of nosocomial COVID-19 infection in patients or HCPs is therefore essential for the setting up of immediate investigation and implementation of appropriate hygiene measures. Describing the signs and symptoms associated with nosocomial COVID-19 will enable comparisons with existing data on other nosocomial viral respiratory infections (ie, influenza, RSV, SARS-CoV and MERS-CoV).

The prospective design and face-to-face interviews will make it possible to reduce recall bias and to collect accurate data. The expertise of the Lyon University-affiliated hospitals already strongly involved in the surveillance of nosocomial infections and the extensive experience of GABRIEL network countries in various research studies related to respiratory infections also strengthen this research project. Participation in this project will enable the subjects to benefit from the diagnostic results. Moreover, each centre will benefit from the overall data in order to explore a particular scientific theme. The multicentric approach will allow high power for this study and enable comparison of clinical characteristics of COVID-19 in worldwide settings.

The results of the Nosocomila transmission of coronavirus SARS-CoV-2 (NOSO-COR) project will provide original results that could (1) constitute additional evidence for a better understanding of the duration of the incubation and contagious period of SARS-CoV-2; (2) make it possible to tailor the definition of nosocomial SARS-CoV-2 infection; (2) strengthen preventive campaigns for in-hospital transmission of SARS-CoV-2; (3) pave the way for new recommendations in terms of preventive measures; (4) supplement existing recommendations by acquiring additional data concerning the transmission of the virus and thereby contributing to improving control guidelines for similar respiratory viral epidemics; (5) identify subpopulations that are at risk of acquiring COVID-19 at both community and hospital levels.

Communication of the results of this study could raise awareness among HCPs vis-à-vis their roles in preventing the spread of the virus in hospitals and in their immediate surroundings and could be used to support vaccination coverage if a vaccine becomes available in the future. The contribution of strain genotyping, when these data become available on a large scale, could complement epidemiological investigations targeting nosocomial transmission of COVID-19. ${ }^{26}$

Asymptomatic and paucisymptomatic cases will not be included in this study, although SARS-CoV-2 transmission from asymptomatic cases has been documented. ${ }^{27}$ This represents a major weakness of our study because asymptomatic individuals may not be recognised by healthcare workers, although they can become the source of nosocomial transmission in the hospital. Furthermore, there is a risk of missing even symptomatic cases owing to the intensity of the outbreak. This protocol has been drafted at the early stage of the epidemic. Data might change over time. As many hospitals in France and overseas will be included, reporting bias may be existing according to the availability of the data across the participating facilities. A measurement bias on the laboratory parameters may occur as the laboratory tests are not performed by a centralised testing facility.

\section{Author affiliations}

${ }^{1}$ Laboratoire des Pathogènes Emergents, Fondation Mérieux, Centre International de Recherche en Infectiologie (CIRI), INSERM U1111, CNRS, Lyon, France

${ }^{2}$ Service Hygiène, Epidémiologie et Prévention, Centre Hospitalier Edouard Herriot, Hospices Civils de Lyon, Lyon, France

${ }^{3}$ Fondation Merieux, Lyon, Rhône-Alpes, France

${ }^{4}$ Laboratoire de Virologie, Institut des Agents Infectieux, Hôpital de la Croix-Rousse, Hospices Civils de Lyon, Lyon, France

${ }^{5}$ Virpath, Grippe, de l'émergence au contrôle, Centre International de Recherche en Infectiologie (CIRI), Inserm U111, CNRS 5308, ENS, UCBL1, Faculté de Médecine RTH Laënnec, Lyon, France

${ }^{6}$ Inserm, F-CRIN, Lyon center of Innovative Clinical Research Network in Vaccinology (I REIVAC), CIC 1417, Paris, France 
Acknowledgements The authors thank M Peter Tucker for editing the manuscript and Marie Moroso, Cindy Grasso and Melina Messaoudi for their involevement in the set-up of the study in GABRIEL network countries.

Contributors PV designed the study. MS-E, VP, LH, FKP, VE, CD, CE, HPE and PV participated in the design of the CRF, drafting and revision of the protocol and manuscript, and approved the final version.

Funding The authors have not declared a specific grant for this research from any funding agency in the public, commercial or not-for-profit sectors.

Competing interests None declared.

Patient consent for publication Not required.

Provenance and peer review Not commissioned; externally peer reviewed.

Supplemental material This content has been supplied by the author(s). It has not been vetted by BMJ Publishing Group Limited (BMJ) and may not have been peer-reviewed. Any opinions or recommendations discussed are solely those of the author(s) and are not endorsed by BMJ. BMJ disclaims all liability and responsibility arising from any reliance placed on the content. Where the content includes any translated material, BMJ does not warrant the accuracy and reliability of the translations (including but not limited to local regulations, clinical guidelines, terminology, drug names and drug dosages), and is not responsible for any error and/or omissions arising from translation and adaptation or otherwise.

Open access This is an open access article distributed in accordance with the Creative Commons Attribution Non Commercial (CC BY-NC 4.0) license, which permits others to distribute, remix, adapt, build upon this work non-commercially, and license their derivative works on different terms, provided the original work is properly cited, appropriate credit is given, any changes made indicated, and the use is non-commercial. See: http://creativecommons.org/licenses/by-nc/4.0/.

\section{ORCID iD}

Mitra Saadatian-Elahi http://orcid.org/0000-0003-1265-8806

\section{REFERENCES}

1 de Wit E, van Doremalen N, Falzarano D, et al. SARS and MERS: recent insights into emerging coronaviruses. Nat Rev Microbiol 2016;14:523-34.

2 Ji W, Wang W, Zhao X, et al. Cross-species transmission of the newly identified coronavirus 2019-nCoV. J Med Virol 2020;92:433-40.

3 Hui DS, I Azhar E, Madani TA, et al. The continuing 2019-nCoV epidemic threat of novel coronaviruses to global health - The latest 2019 novel coronavirus outbreak in Wuhan, China. Int J Infect Dis 2020:91:264-6.

4 Zhu N, Zhang D, Wang W, et al. A novel coronavirus from patients with pneumonia in China, 2019. N Engl J Med 2020;382:727-33.

$5 \mathrm{Lu} \mathrm{H}$, Stratton CW, Tang Y-W. Outbreak of pneumonia of unknown etiology in Wuhan, China: the mystery and the miracle. J Med Virol 2020;92:401-2.

6 Li R, Pei S, Chen B, et al. Substantial undocumented infection facilitates the rapid dissemination of novel coronavirus (SARSCoV-2). Science 2020;368:489-93.

7 Jin Y-H, Cai L, Cheng Z-S, et al. A rapid advice guideline for the diagnosis and treatment of 2019 novel coronavirus (2019-nCoV) infected pneumonia (standard version). Mil Med Res 2020;7:4.

8 Lupia T, Scabini S, Mornese Pinna S, Pinna SM, et al. 2019 novel coronavirus (2019-nCoV) outbreak: a new challenge. J Glob Antimicrob Resist 2020;21:22-7.
9 Tian Y, Rong L, Nian W, et al. Review article: gastrointestinal features in COVID-19 and the possibility of faecal transmission. Aliment Pharmacol Ther 2020;51:843-51.

10 Adhikari SP, Meng S, Wu Y-J, et al. Epidemiology, causes, clinical manifestation and diagnosis, prevention and control of coronavirus disease (COVID-19) during the early outbreak period: a scoping review. Infect Dis Poverty 2020;9:29.

$11 \mathrm{Hu} Z$, Song C, Xu C, et al. Clinical characteristics of 24 asymptomatic infections with COVID-19 screened among close contacts in Nanjing, China. Sci China Life Sci 2020;63:706-11.

12 Santé Publique France. Coronavirus: chiffres clés et évolution de la COVID-19 en France et dans Le Monde. Available: https://www. santepubliquefrance.fr/dossiers/coronavirus-covid-19/coronaviruschiffres-cles-et-evolution-de-la-covid-19-en-france-et-dans-lemonde\#block-266151

13 Lauer SA, Grantz KH, Bi Q, et al. The incubation period of coronavirus disease 2019 (COVID-19) from publicly reported confirmed cases: estimation and application. Ann Intern Med 2020;172:577-82.

14 Huang C, Wang Y, Li X, et al. Clinical features of patients infected with 2019 novel coronavirus in Wuhan, China. Lancet 2020;395:497-506.

15 Centre for Disease Control. Interim infection prevention and control recommendations for patients with known or patients under investigation for 2019 novel coronavirus (2019-nCoV) in a healthcare setting. Available: https://www.cdc.gov/coronavirus/2019-nCoV/hcp/ infection-control.html

16 World Health Organisation. Infection prevention and control during health care when novel coronavirus (nCoV) infection is suspected interim guidance, 2020.

17 Wang D, Hu B, Hu C, et al. Clinical characteristics of 138 hospitalized patients with 2019 novel coronavirus-infected pneumonia in Wuhan, China. JAMA 2020;323:1061.

18 The Lancet. COVID-19: protecting health-care workers. Lancet 2020;395:922.

19 Santé Publique France. Recensement national des Cas de COVID-19 CheZ les professionnels en établissements de santé. Available: https://www.santepubliquefrance.fr/etudes-et-enquetes/ recensement-national-des-cas-de-covid-19-chez-les-professionnelsen-etablissements-de-sante

20 Voirin N, Payet C, Barrat A, et al. Combining high-resolution contact data with virological data to investigate influenza transmission in a tertiary care hospital. Infect Control Hosp Epidemiol 2015;36:254-60.

21 Vanhems P, Barrat A, Cattuto C, et al. Estimating potential infection transmission routes in hospital wards using wearable proximity sensors. PLoS One 2013;8:e73970.

22 Vanhems P, Voirin N, Bénet T, et al. Detection of hospital outbreaks of influenza-like illness based on excess of incidence rates compared to the community. Am J Infect Control 2014;42:1325-7.

23 Munier-Marion E, Bénet T, Régis C, et al. Hospitalization in doubleoccupancy rooms and the risk of hospital-acquired influenza: a prospective cohort study. Clin Microbiol Infect 2016;22:461.e7-9.

24 World Health Organisation. Coronavirus disease 2019 (COVID-19) situation report - 70. page 9. Available: https://www.who.int/docs/ default-source/coronaviruse/situation-reports/20200330-sitrep-70covid-19.pdf?sfvrsn=7e0fe3f8_2

25 International Committee of Medical Journal Editors. Uniform requirements for manuscripts submitted to biomedical journals. $N$ Engl J Med 1997;336:309-16.

26 Chen Y, Liu Q, Guo D. Emerging coronaviruses: genome structure, replication, and pathogenesis. J Med Virol 2020;92:418-23.

27 Tian S, Hu N, Lou J, et al. Characteristics of COVID-19 infection in Beijing. J Infect 2020;80:401-6. 